\title{
PERFIS DE DISTRIBUIÇÃO VOLUMÉTRICA DE PONTAS XR 11003 E TXVK-4 EM DIFERENTES CONDIÇÕES DE PULVERIZAÇÃO
}

\author{
FERNANDO C. BAUER ${ }^{1}$, CARLOS G. RAETANO ${ }^{2}$
}

RESUMO: Com o objetivo de avaliar a uniformidade da distribuição volumétrica e o efeito da angulação dos bicos na barra em pulverizações, foi conduzido experimento em laboratório, com dez unidades de cada uma das pontas de jato plano XR 11003 e de jato cônico vazio TXVK-4, fabricadas pela Spraying Systems Co. As pontas XR 11003 foram avaliadas, isoladamente, em mesa de prova para bicos construída conforme a Norma ISO 5682-1:1996, a 0,30; 0,40 e 0,50 m de altura, submetidas à pressão de 200 e $300 \mathrm{kPa}$ e posicionadas com ângulos de $30^{\circ} \mathrm{e} 45^{\circ}$ no sentido horário e anti-horário e na vertical; as pontas TXVK-4 foram avaliadas nas mesmas alturas, porém a 300 e $400 \mathrm{kPa}$, e somente na vertical. A distribuição média do volume coletado em cada configuração, das dez unidades de cada ponta, foi inserida num programa computacional, desenvolvido para o estudo, que permitiu a simulação do padrão de deposição de uma barra pulverizadora, com 24 bicos, com sobreposições variáveis, obtendo-se, ao final, o coeficiente de variação da sobreposição, o desvio- padrão e a média do volume aplicado. Os resultados sugerem a necessidade de ajustes no espaçamento entre bicos para a obtenção de melhor uniformidade na distribuição, principalmente quando se utiliza ângulo na barra; há menor influência da variação da altura da barra sobre a uniformidade da distribuição quando o espaçamento entre bicos é reduzido.

PALAVRAS-CHAVE: ponta de pulverização, coeficiente de variação, ângulo de pulverização.

\section{SPRAY DISTRIBUTION PATTERNS OF THE XR 11003 AND TXVK-4 HYDRAULIC NOZZLES UNDER DIFFERENT CONDITIONS}

SUMMARY: The objective of this study was to evaluate the uniformity of spray distribution as well as the angle effect of hydraulic nozzles in a spray boom. Ten units of flat fan nozzle XR 11003 and hollow cone TXVK-4 (Spraying Systems Co) were tested in a patternator table built in accordance to the standard ISO 5682-1:1996. Each one of the units was tested separately, at the height of 0.30, 0.40 and $0.50 \mathrm{~m}$, at 200 and $300 \mathrm{kPa}$ and angles of $30^{\circ}$ and $45^{\circ}$, clockwise and counterclockwise and in the vertical position. TXVK-4 nozzles were tested at same heights but at 300 and $400 \mathrm{kPa}$ and only in the vertical position. The mean of the collected spray volume distribution of each nozzle type tip was entered in a computer program developed. This program simulated the distribution pattern of 24 nozzles boom at different overlapping and calculated the coefficient of variation corresponding to each configuration. The results pointed out to the necessity of the adaptation in nozzles spacing especially if any angle is in use and that the distribution uniformity is less vulnerable to boom height variation at reduced nozzle spacing.

KEYWORDS: nozzles, coefficient of variation, spray angle.

\footnotetext{
${ }^{1}$ Engo . Agrônomo, Doutorando do Curso de Pós-Graduação em Agronomia (Proteção de Plantas), Faculdade de Ciências Agronômicas, UNESP, Botucatu - SP, e-mail: fernandobauer@fca.unesp.br, Bolsista FAPESP.

${ }^{2}$ Eng ${ }^{\mathrm{o}}$. Agronômo, Prof. Dr., Departamento de Produção Vegetal - Defesa Fitossanitária, Faculdade de Ciências Agronômicas, UNESP, Botucatu - SP.

Recebido pelo Conselho Editorial em: 25-9-2002

Aprovado pelo Conselho Editorial em: $1^{\underline{0}}-12-2003$
} 


\section{INTRODUÇÃO}

É função do equipamento de aplicação a distribuição da pulverização de forma correta sobre o alvo. Muitos são os fatores que interferem negativamente na qualidade da pulverização, dentre os quais, os mais importantes são a calibração inadequada do equipamento, a falta de controle do tamanho e da distribuição das gotas pulverizadas, o volume de aplicação inadequado, a má cobertura sobre o alvo, a altura inadequada da barra pulverizadora, a época de aplicação e a escolha incorreta dos bicos (SHARP, 1973).

DOWNER et al. (1997) afirmam que o objetivo de muitos pesquisadores é reduzir a quantidade de produto aplicado. Com doses e volumes reduzidos, a uniformidade na distribuição deve ser alcançada, caso contrário, a dose que chega ao alvo poderá ser insuficiente para causar o efeito biológico desejável. A uniformidade de deposição do líquido pulverizado e o tamanho das gotas são fatores que dependem do desempenho do órgão emissor de gotas que, no caso dos pulverizadores, é o bico hidráulico, considerado a parte mais importante do equipamento (GALLI \& ARRUDA, 1985).

Uma das formas de quantificar a uniformidade de distribuição numa pulverização é por meio da análise da deposição do produto na área, que é expressa pelo coeficiente de variação (C.V.) obtido dessa análise; quanto menor esse valor, mais uniforme é a distribuição (CHRISTOFOLETTI, 1997). Assim, segundo FURLANETTI (1998), um C.V. de 0\% indica que um mesmo volume foi aplicado ao longo de toda a barra de pulverização, ou seja, distribuição com uniformidade absoluta.

MATTHEWS (1992) afirma que cada bico possui uma característica própria de distribuição volumétrica e que essa curva tem grande importância na determinação da altura do bico em relação ao alvo e no espaçamento entre bicos na barra, devendo haver sobreposição do jato de um bico com os adjacentes para conseguir distribuição uniforme do líquido pulverizado.

Normalmente, o C.V. é calculado a partir do padrão de distribuição de bicos individuais, simulando-se, então, a sobreposição desses padrões, com exceção de testes oficiais. Assim, os padrões de deposição individual dos bicos são determinados em bancadas de prova, onde canaletas realizam a coleta do líquido a distâncias determinadas e o depositam em recipientes individuais (CHAPPLE et al., 1993).

Assim, o presente trabalho teve como objetivos determinar, em laboratório, quais espaçamentos e alturas de barra produzem melhor distribuição da pulverização, para diferentes tipos de pontas de pulverização, em duas pressões e em diferentes ângulos de aplicação.

\section{MATERIAL E MÉTODOS}

O trabalho foi desenvolvido no Laboratório de Tecnologia de Aplicação de Defensivos Agrícolas do Departamento de Produção Vegetal - Defesa Fitossanitária, da Faculdade de Ciências Agronômicas da Universidade Estadual Paulista, Câmpus de Botucatu.

Uma mesa para testes com bicos hidráulicos foi construída de acordo com a Norma ISO 5.682-1:1996. A mesa consta de 60 canaletas de alumínio de $50 \mathrm{~mm}$ de largura e $2.000 \mathrm{~mm}$ de comprimento cada, totalizando $3.000 \mathrm{~mm}$ x $2.000 \mathrm{~mm}$ de área útil de coleta. As canaletas possuem desnível de 10\%, sendo a altura do fundo de $12 \mathrm{~mm}$ e na frente $32 \mathrm{~mm}$. Ao final de cada canaleta, conectou-se um tubo plástico de drenagem para conduzir o líquido a uma proveta graduada e numerada, de acordo com a canaleta a que está ligada.

A barra pulverizadora, em que os bicos foram fixados, é independente da mesa, em forma de "U“ invertido, com o travessão ficando a $2.300 \mathrm{~mm}$ de altura, porém sendo possível sua regulagem a cada $50 \mathrm{~mm}$, podendo-se trabalhar com a barra desde 100 até $1.500 \mathrm{~mm}$ de altura em relação à superfície da mesa. Na própria barra pulverizadora, há um dispositivo circular que permite incliná-la, em intervalos de $5^{\circ}$ por até $90^{\circ}$ em relação à vertical, tanto no sentido horário quanto no anti-horário. 
Na parte central da barra pulverizadora foi fixado um manômetro, com precisão de $0,5 \%$, do qual se monitorou a pressão a que as pontas em teste foram submetidas. O sistema funciona com energia proveniente de motor elétrico trifásico com potência de $2,98 \mathrm{~kW}(4 \mathrm{cv})$ que, por meio de correias, movimenta uma bomba de três êmbolos marca Jacto, com vazão de $42 \mathrm{~L} \mathrm{~min}^{-1}$ e pressão máxima de $3.431 \mathrm{kPa}$.

Foram adquiridas, junto a um distribuidor da Spraying System Co., dez unidades das pontas de pulverização XR 11003, de jato plano, e TXVK-4, de jato cônico vazio. Cada unidade da ponta XR 11003 foi instalada, isoladamente, nas alturas de 0,$30 ; 0,40$ e $0,50 \mathrm{~m}$ em relação à superfície da mesa e, em cada altura, submetidas às pressões de 200 e $300 \mathrm{kPa}$. Para cada altura e pressão em teste, foi colocada a respectiva ponta na posição vertical $\left(0^{\circ}\right)$ e nos ângulos de 30 e $45^{\circ}$ no sentido horário e antihorário. A ponta cônica TXVK-4 foi submetida às mesmas alturas e a pressões de 300 e $400 \mathrm{kPa}$, sendo utilizada somente na posição vertical. Em todos os experimentos, o líquido utilizado para as pulverizações foi água proveniente da rede pública à temperatura ambiente.

Assim, cada ponta foi instalada centrada na mesa e posta em funcionamento por período igual a dois minutos, visando à estabilização no fluxo do líquido nas canaletas; após esse período, era feita a coleta do líquido pulverizado por um minuto quando, então, se encerrava a coleta e se fazia a leitura dos volumes contidos em cada proveta.

Ao considerar o padrão médio de distribuição volumétrica de cada tipo de ponta, para cada condição operacional, iniciaram-se os estudos de sobreposição, individualmente, para cada uma das configurações. Para isso, foi desenvolvido um programa computacional, na Faculdade de Ciências Agrárias e Veterinárias da UNESP - Câmpus de Jaboticabal, similar ao utilizado por PERECIN et al. (1994), que, a partir de pontas individuais, simula a deposição da pulverização em toda a barra. O programa funciona de forma independente da configuração, considerando o número de canaletas fornecido e o número de canaletas que serão sobrepostas, possibilitando a simulação de uma barra hipotética, em que se pode trabalhar com um número infinito de bicos e com sobreposições variáveis.

Ao final das análises, o programa fornece o coeficiente de variação (C.V.) do resultado da sobreposição, o desvio-padrão e a média do volume aplicado em mililitros.

Com relação à simulação dos bicos em barra total, PERECIN et al. (1998) indicam ser esse procedimento aceitável do ponto de vista estatístico, uma vez que a causa das variabilidades sistemática (CVS) e aleatória (CVA) é dependente de causas diferentes. Por isso, neste trabalho, serão apresentados os valores relativos ao C.V. resultante $(\mathrm{CVG})$ dos dois anteriores, calculados pela equação abaixo, por eles proposta.

$$
\mathrm{CVG}=\left[(\mathrm{CVA})^{2}+(\mathrm{CVS})^{2}\right]^{1 / 2}
$$

\section{RESULTADOS E DISCUSSÃO}

O volume coletado em cada canaleta da mesa de teste permitiu avaliar o perfil de distribuição volumétrico característico de cada ponta e, com o auxílio do programa computacional, foi feita a simulação da sobreposição, individualmente, para todas as configurações em teste, de uma barra de pulverização de 24 bicos, variando-se a sobreposição de $5 \mathrm{em} 5 \mathrm{~cm}$. Os dados de deposição foram utilizados sob a forma de porcentagem do volume aplicado.

Do ponto de vista prático, o coeficiente de variação (C.V.) sob a barra é mais importante que o volume depositado, pois poderão existir diferentes volumes de depósito com valores de C.V. idênticos; por isso, neste trabalho, optou-se por avaliar somente o C.V., independentemente de outras variáveis. Isso se deu em função do grande número de configurações (36) e da grande diferença entre elas (Tabela 1). Além disso, as vazões médias obtidas apresentaram C.V. ao redor de $3 \%$, indicando boa repetibilidade para esse parâmetro. 
A utilização do programa computacional resultou em grande quantidade de informação, impossibilitando a apresentação de todos os dados. Isso ocorreu devido ao fato de, em cada configuração, ter sido feita a simulação da sobreposição, a cada $5 \mathrm{~cm}$, até a largura total da deposição. Exemplificando, para a ponta XR 11003 avaliada a $0,30 \mathrm{~m}$ de altura, a $200 \mathrm{kPa}$ e instalada na posição vertical, resultou numa deposição total de $110 \mathrm{~cm}$ e 21 sobreposições, em 21 espaçamentos diferentes. $\mathrm{Na}$ Tabela 1, apresentam-se as configurações utilizadas e seus resultados, indicando a largura total da deposição, o maior espaçamento entre bicos, cuja simulação obteve C.V. imediatamente inferior a $10 \%$, e a porcentagem da sobreposição numa barra simulada de 24 bicos.

TABELA 1. Faixa da deposição $(\mathrm{cm})$, espaçamento entre bicos e porcentagem de sobreposição obtidos em pulverizações com C.V. abaixo de $10 \%$, indicando tipos de ponta, pressão, altura da barra e ângulo dos bicos utilizados na avaliação da distribuição da pulverização.

\begin{tabular}{|c|c|c|c|c|c|c|}
\hline $\begin{array}{c}\text { Ponta } \\
\text { Utilizada }\end{array}$ & $\begin{array}{l}\text { Altura } \\
(\mathrm{m})\end{array}$ & $\begin{array}{c}\text { Pressão } \\
(\mathrm{kPa})\end{array}$ & $\begin{array}{l}\text { Ângulo/ } \\
\text { Sentido }\end{array}$ & $\begin{array}{l}\text { Largura da Faixa de } \\
\text { Deposição Total }(\mathrm{cm})\end{array}$ & $\begin{array}{l}\text { Espaçamento com } \\
\text { C.V. }<10 \%(\mathrm{~cm})\end{array}$ & $\begin{array}{c}\text { Sobreposição } \\
(\%)\end{array}$ \\
\hline \multirow{30}{*}{ XR 11003} & \multirow{10}{*}{0,30} & \multirow{5}{*}{200} & vertical & 110 & 35 & 68 \\
\hline & & & $30^{\circ}$ horário & 130 & 50 & 62 \\
\hline & & & $30^{\circ}$ anti-horário & 110 & 40 & 64 \\
\hline & & & $45^{\circ}$ horário & 160 & 80 & 50 \\
\hline & & & $45^{\circ}$ anti-horário & 130 & 55 & 58 \\
\hline & & \multirow{5}{*}{300} & vertical & 120 & 40 & 67 \\
\hline & & & $30^{\circ}$ horário & 145 & 55 & 62 \\
\hline & & & $30^{\circ}$ anti-horário & 120 & 40 & 67 \\
\hline & & & $45^{\circ}$ horário & 175 & 85 & 51 \\
\hline & & & $45^{\circ}$ anti-horário & 140 & 50 & 64 \\
\hline & \multirow{10}{*}{0,40} & \multirow{5}{*}{200} & vertical & 140 & 50 & 64 \\
\hline & & & $30^{\circ}$ horário & 170 & 80 & 53 \\
\hline & & & $30^{\circ}$ anti-horário & 145 & 55 & 62 \\
\hline & & & $45^{\circ}$ horário & 195 & 105 & 46 \\
\hline & & & $45^{\circ}$ anti-horário & 160 & 80 & 50 \\
\hline & & \multirow{5}{*}{300} & vertical & 150 & 55 & 63 \\
\hline & & & $30^{\circ}$ horário & 180 & 80 & 56 \\
\hline & & & $30^{\circ}$ anti-horário & 160 & 60 & 63 \\
\hline & & & $45^{\circ}$ horário & 200 & 115 & 43 \\
\hline & & & $45^{\circ}$ anti-horário & 180 & 70 & 61 \\
\hline & \multirow{10}{*}{0,50} & \multirow{5}{*}{200} & vertical & 170 & 65 & 62 \\
\hline & & & $30^{\circ}$ horário & 190 & 100 & 47 \\
\hline & & & $30^{\circ}$ anti-horário & 170 & 85 & 50 \\
\hline & & & $45^{\circ}$ horário & 210 & 125 & 40 \\
\hline & & & $45^{\circ}$ anti-horário & 185 & 95 & 49 \\
\hline & & \multirow{5}{*}{300} & vertical & 185 & 70 & 62 \\
\hline & & & $30^{\circ}$ horário & 200 & 110 & 45 \\
\hline & & & $30^{\circ}$ anti-horário & 185 & 85 & 54 \\
\hline & & & $45^{\circ}$ horário & 220 & 135 & 39 \\
\hline & & & $45^{\circ}$ anti-horário & 195 & 110 & 44 \\
\hline \multirow{6}{*}{ TXVK-4 } & \multirow{2}{*}{0,30} & 300 & vertical & 50 & 30 & 40 \\
\hline & & 400 & vertical & 50 & 30 & 40 \\
\hline & \multirow{2}{*}{0,40} & 300 & vertical & 55 & 35 & 36 \\
\hline & & 400 & vertical & 60 & 30 & 50 \\
\hline & \multirow{2}{*}{0,50} & 300 & vertical & 65 & 30 & 54 \\
\hline & & 400 & vertical & 65 & 35 & 46 \\
\hline
\end{tabular}


Analisando as pontas TXVK-4, pode-se observar que a altura da barra e a pressão não tiveram influência no espaçamento e no C.V., mas a altura da barra teve influência direta na largura da deposição (Figura 1), mas não no espaçamento entre bicos, pois os espaçamentos, com CVs abaixo de $10 \%$, estiveram sempre próximos de $0,30 \mathrm{~m}$, indicando ser essa a distância ideal para essas pontas. O comportamento do C.V., em relação aos espaçamentos testados, pode ser observado na Figura 2. Para espaçamentos entre bicos superiores a $0,35 \mathrm{~m}$, quanto menor a altura, maior o C.V., tornando essa relação mais evidente na maior pressão em teste.

Os padrões de distribuição das pontas XR 11003, para as diferentes alturas e pressões utilizadas, encontram-se na Figura 3. Pode-se observar grande influência na largura da distribuição quando se posicionam os bicos na barra com algum ângulo. $\mathrm{O}$ uso dessa ponta com $45^{\circ}$ de inclinação, no sentido horário, resultou em aumento na largura da deposição equivalente a essa mesma ponta posicionada na vertical, porém com a barra $10 \mathrm{~cm}$ mais alta. Isso mostra que a angulação dos bicos na barra tem efeito parecido, quando se relaciona à deposição, à elevação da barra, refletindo negativamente na sobreposição. Essa constatação indica que deve haver, necessariamente, adequação do espaçamento entre bicos quando se utiliza qualquer ângulo da barra pulverizadora.
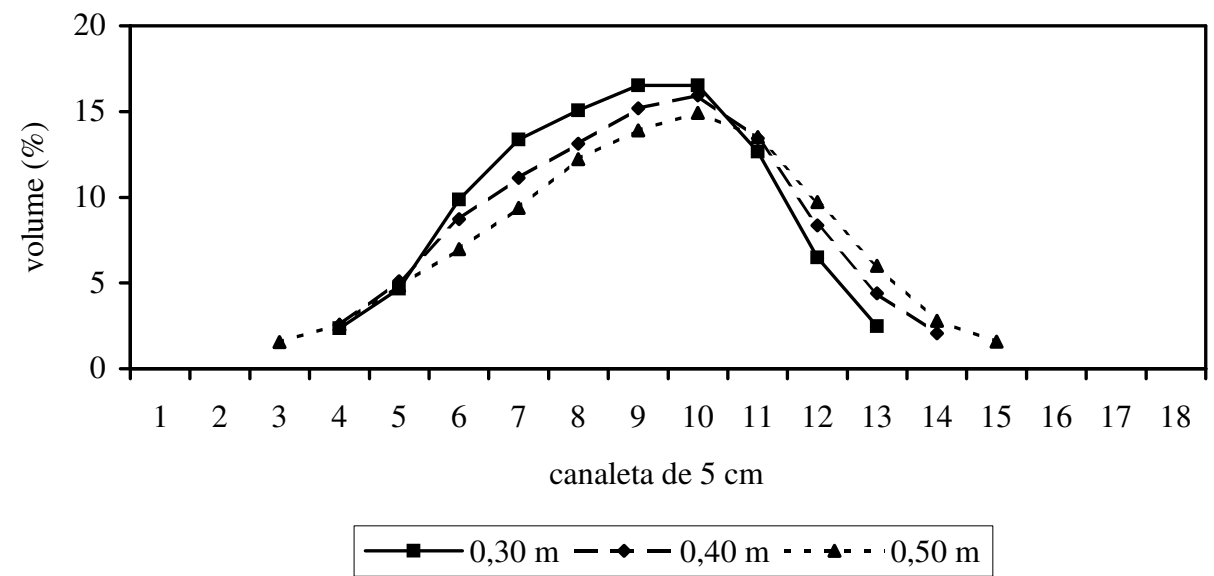

(a)

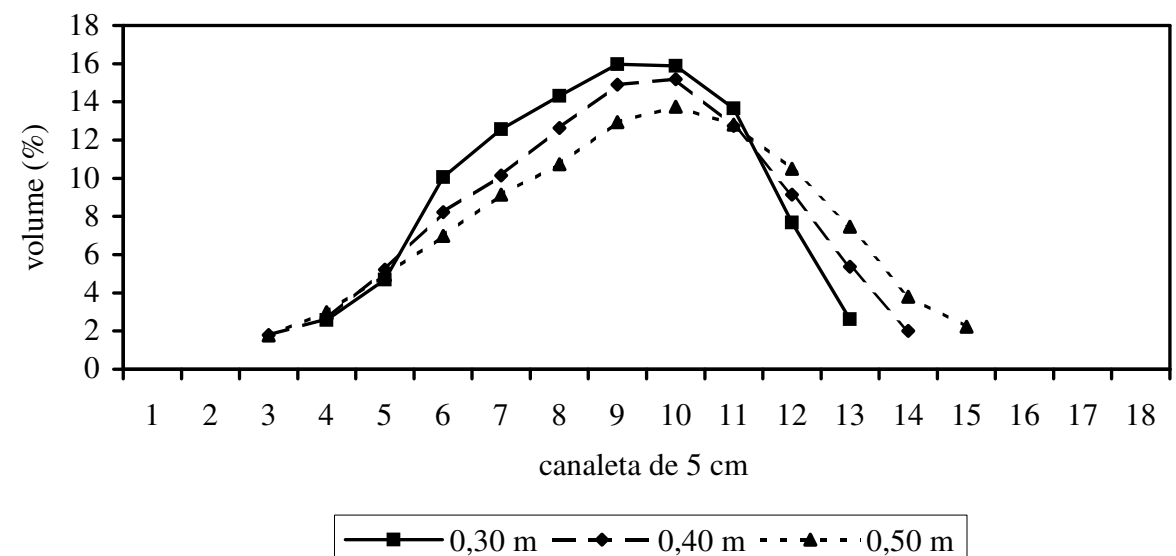

(b)

FIGURA 1. Perfis de deposição médio $(n=10)$ de pontas de jato cônico vazio TXVK-4, a 300 (a) e $400 \mathrm{kPa}(\mathrm{b})$, em três alturas de barra, com distribuição porcentual do volume depositado em cada canaleta de $5 \mathrm{~cm}$, em mesa de teste para pontas de pulverização hidráulica. 


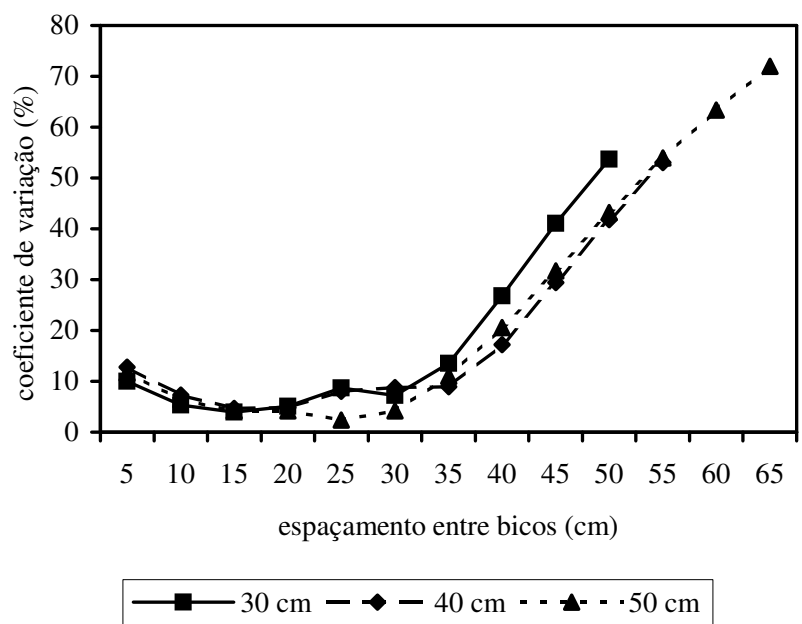

(a)

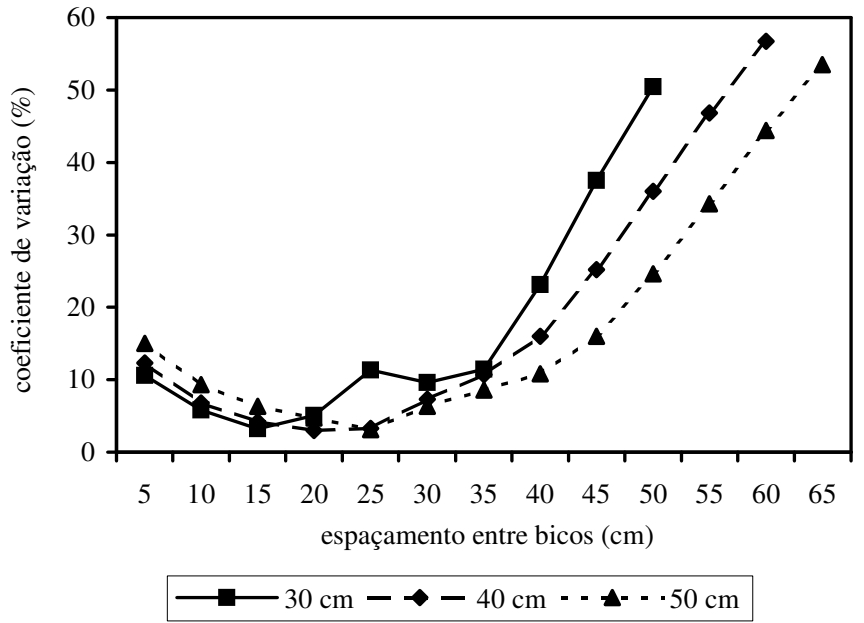

(b)

FIGURA 2. Coeficiente de variação da deposição média $(n=10)$ de pontas de jato cônico vazio TXVK-4 a 300 (a) e $400 \mathrm{kPa}$ (b), em função da variação do espaçamento entre bicos posicionados a 0,$30 ; 0,40$ e $0,50 \mathrm{~m}$ de altura.

Os valores apresentados na Tabela 1 indicam os espaçamentos onde o C.V. da pulverização se situa imediatamente abaixo de $10 \%$. Pode-se observar que, na posição vertical, a recomendação pelo fabricante, de utilização dessa ponta a $40 \mathrm{~cm}$ de altura e de $50 \mathrm{~cm}$ entre bicos, está correta para esses CVs. Porém, ao se adotar C.V. de 7\%, conforme utilizado na Comunidade Européia, esse espaçamento tem de diminuir. Essa observação está de acordo com PERECIN et al. (1994), alertando para a conveniência de se trabalhar com espaçamentos menores que $50 \mathrm{~cm}$.

A observação da Figura 4 reforça essa constatação, pois se o efeito da angulação do bico é o mesmo da elevação da barra, os resultados se mostraram os mesmos, também com relação ao C.V. Esse fato foi demonstrado por AZIMI et al. (1985), com pontas TeeJet 8003.

Os resultados aqui obtidos também indicam ser mais recomendáveis os menores espaçamentos entre bicos, pois há tendência de distribuição mais estável, além de menor influência, por variações de altura e pressão, na uniformidade de distribuição. 


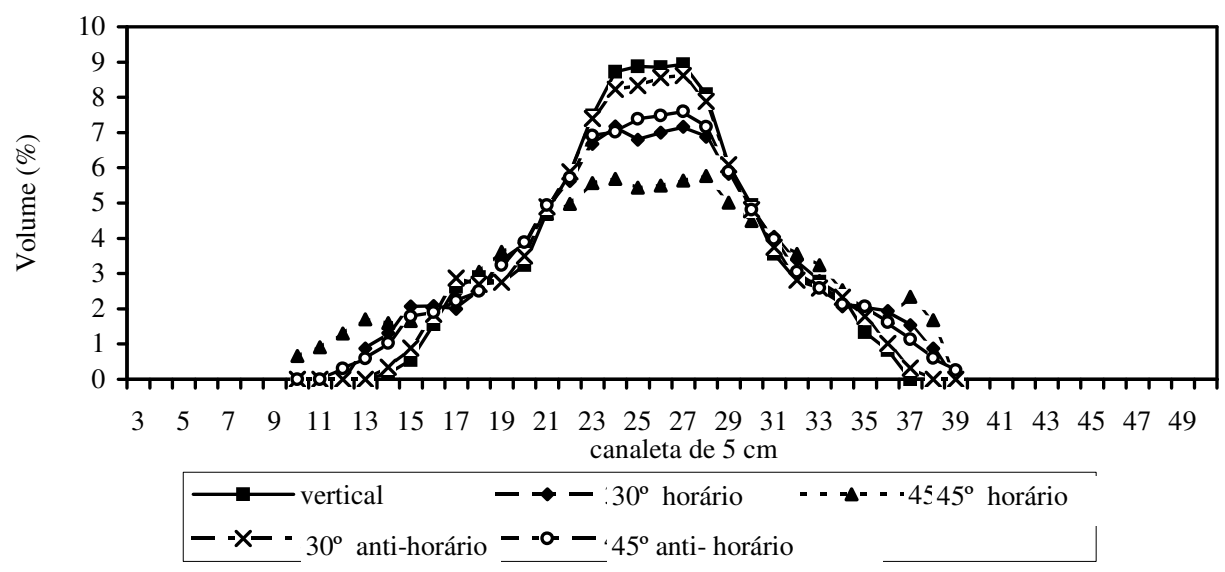

(a)

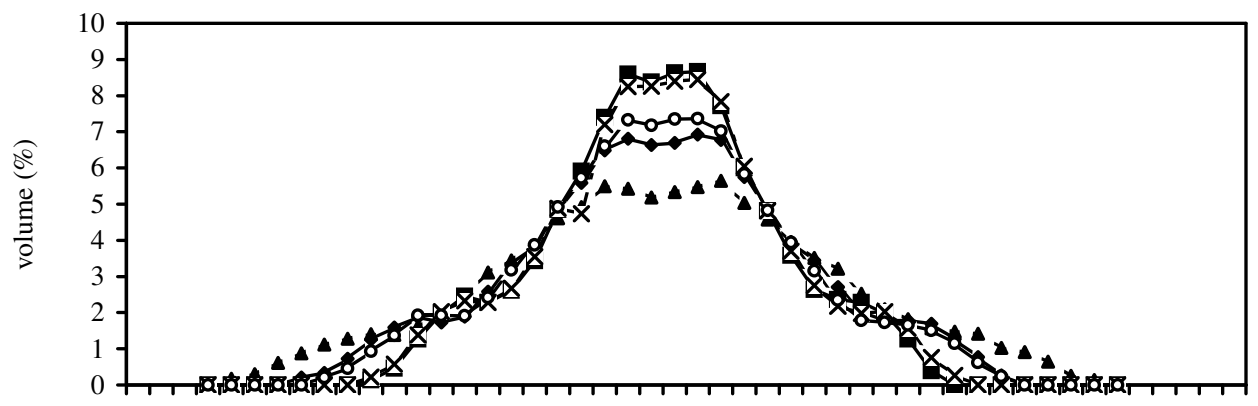

$\begin{array}{llllllllllllllllllllllll}3 & 5 & 7 & 9 & 11 & 13 & 15 & 17 & 19 & 21 & 23 & 25 & 27 & 29 & 31 & 33 & 35 & 37 & 39 & 41 & 43 & 45 & 47 & 49\end{array}$

$\longrightarrow$ vertical $\quad \longrightarrow-: 30^{\circ}$ horário $\quad-\rightarrow-\cdots 4545^{\circ}$ horário

$--x-30^{\circ}$ anti-horário $\quad--\circ-445^{\circ}$ anti-horário

(b)

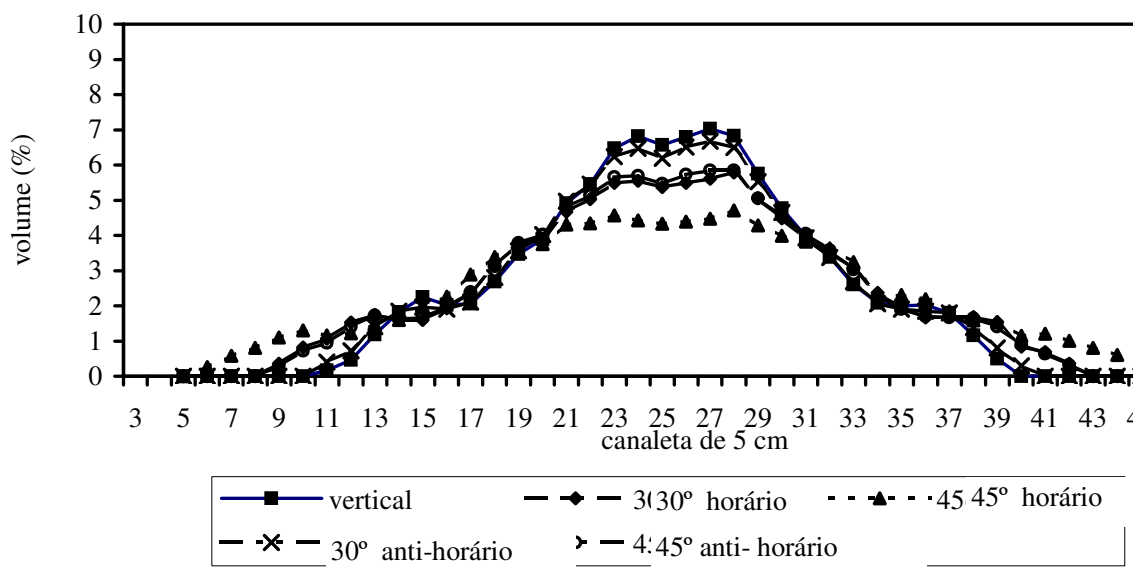

(c) 


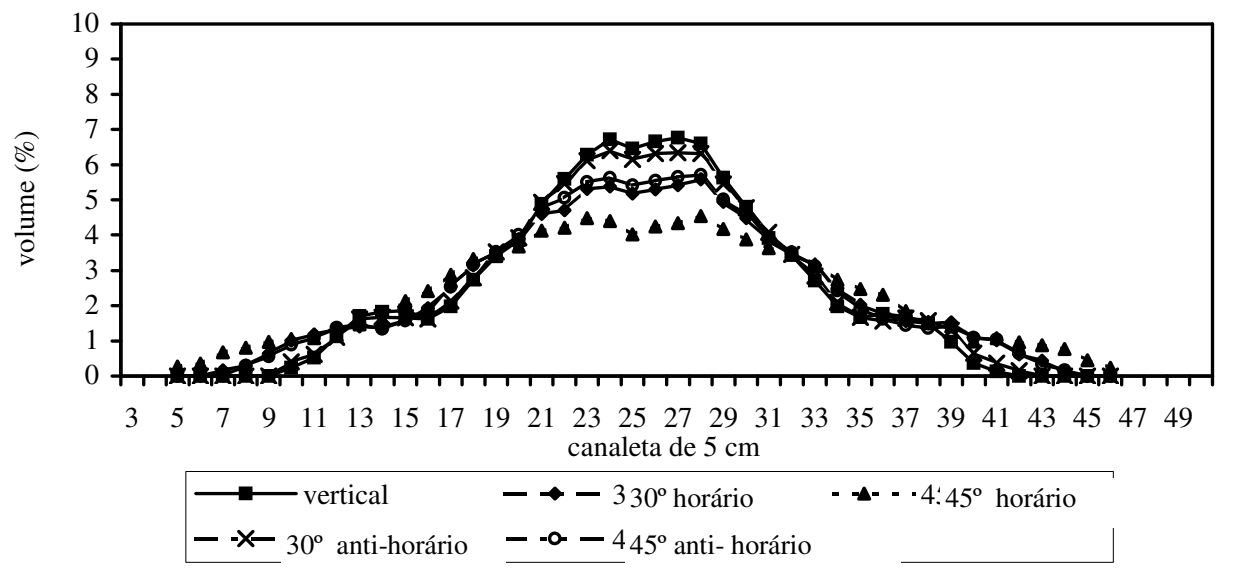

(d)

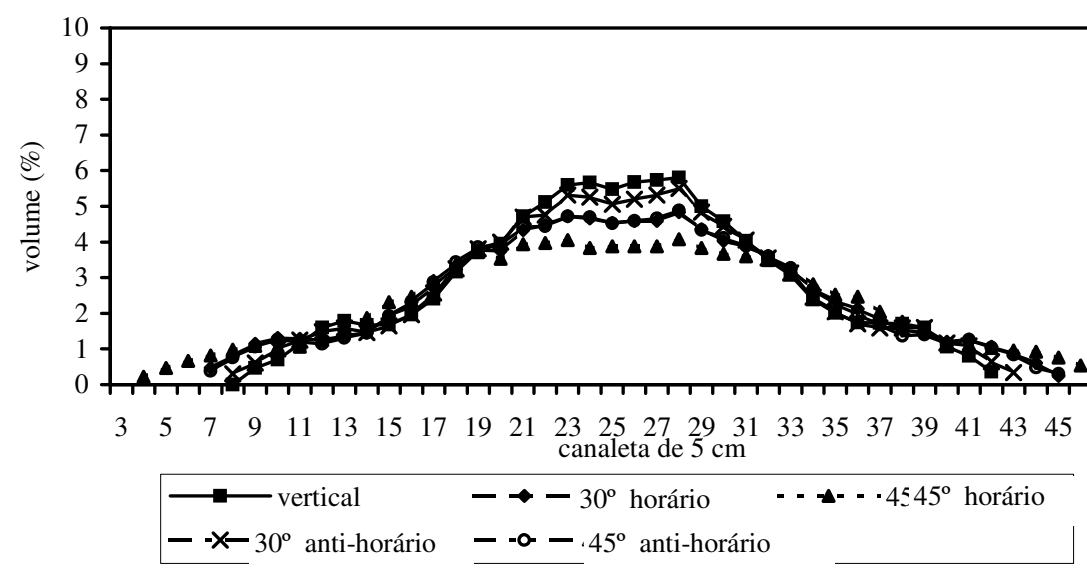

(e)

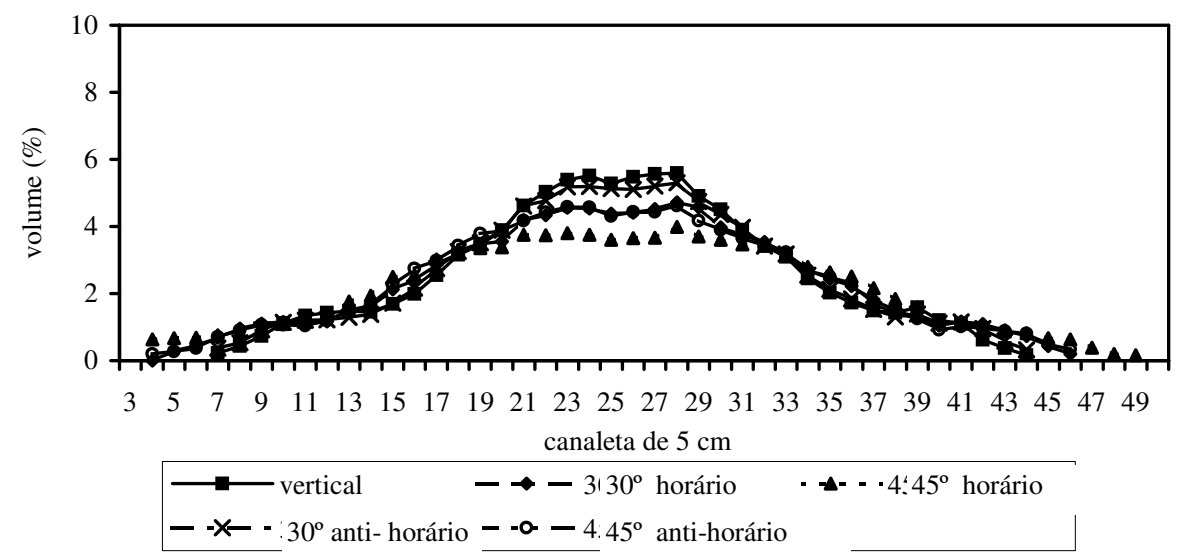

(f)

FIGURA 3. Perfis de deposição médios $(\mathrm{n}=10)$ de pontas de jato plano XR 11003 a $0,30(\mathrm{a}, \mathrm{b}) ; 0,40$ $(\mathrm{c}, \mathrm{d})$ e $0,50 \mathrm{~m}(\mathrm{e}, \mathrm{f})$ de altura e a $200(\mathrm{a}, \mathrm{c}, \mathrm{e})$ e $300 \mathrm{kPa}(\mathrm{b}, \mathrm{d}, \mathrm{f})$, com ângulos de $30^{\circ}$ e $45^{\circ}$ nos sentidos horário e anti-horário e na vertical, com distribuição porcentual do volume depositado em cada canaleta, em mesa de teste para bicos hidráulicos. 


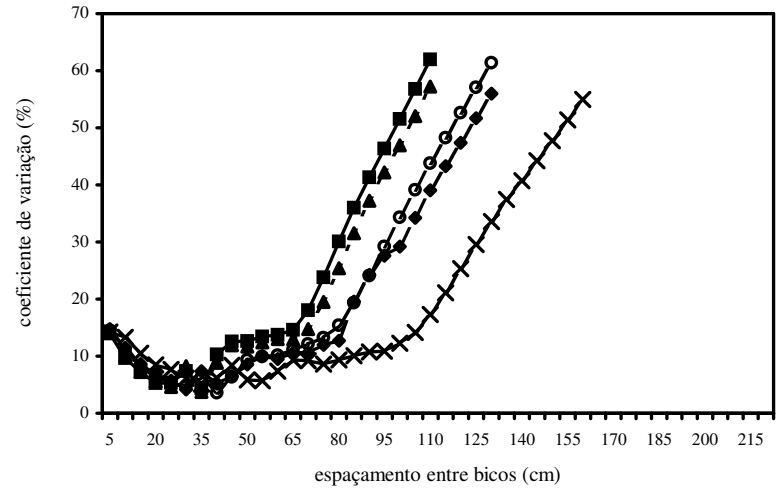

(a)

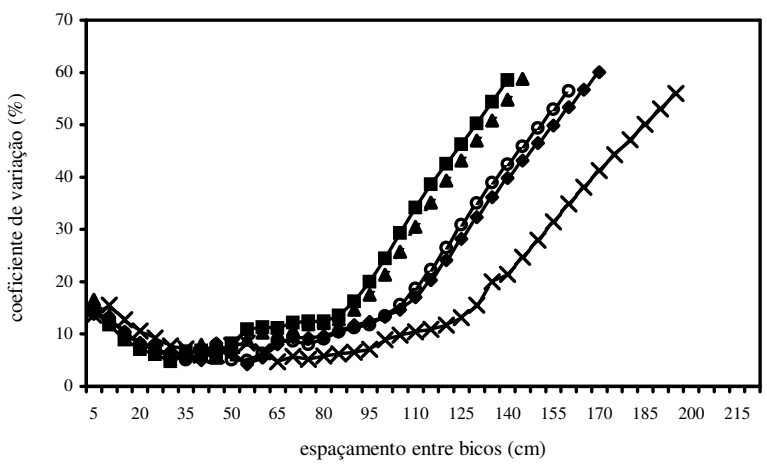

(c)

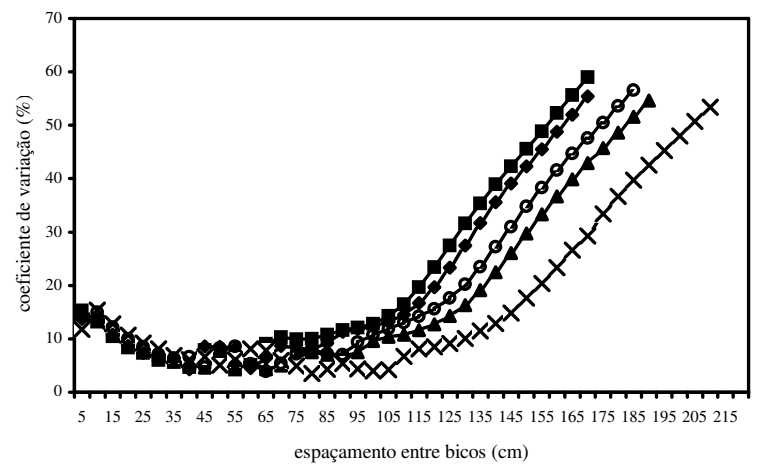

(e)

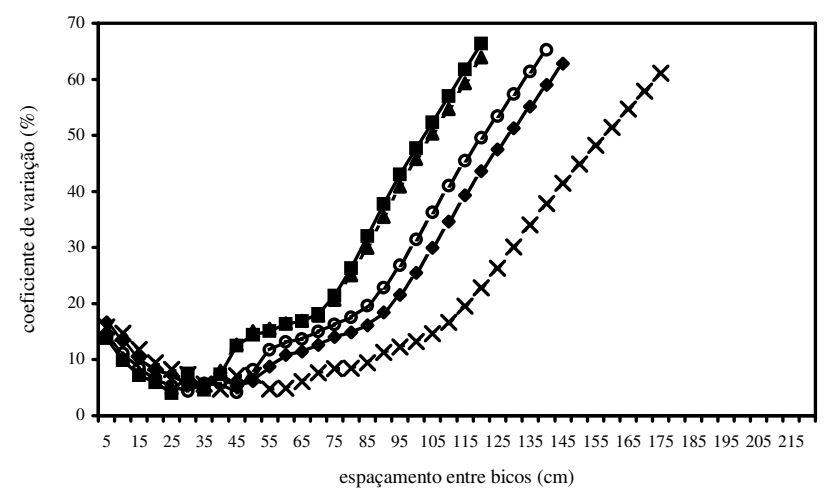

(b)

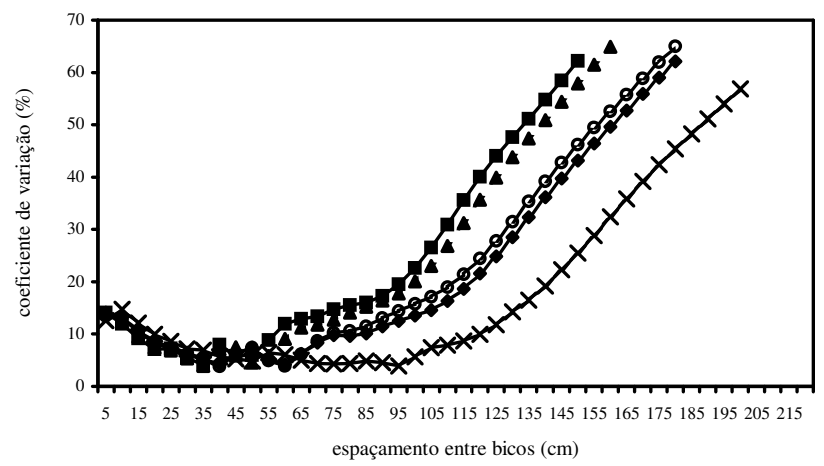

(d)

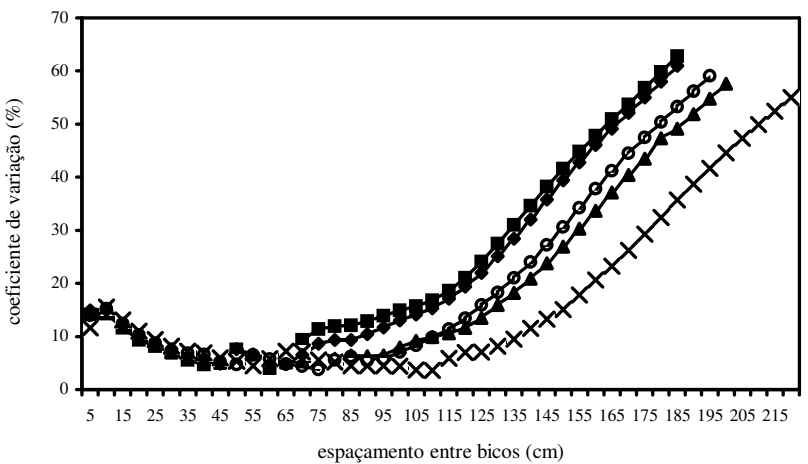

(f)

$\longrightarrow$ vert $\longrightarrow \rightarrow--30 \mathrm{H}---\Delta--30 \mathrm{a}---\times--45 \mathrm{H}--\mathrm{O}--45 \mathrm{~A}$

FIGURA 4. Coeficiente de variação da deposição média $(n=10)$ de pontas de jato plano XR 11003 a 0,30 (a,b); 0,40 (c,d) e 0,50 m (e,f) de altura, a 200 (a,c,e) e $300 \mathrm{kPa}(\mathrm{b}, \mathrm{d}, \mathrm{f})$, em função da variação do espaçamento entre bicos. 


\section{CONCLUSÕES}

A angulação dos bicos na barra pulverizadora tem grande interferência no espaçamento entre bicos, independentemente da pressão de trabalho.

Há a necessidade de maiores estudos relacionados ao espaçamento entre bicos na barra, pois em nenhuma das simulações de sobreposição ocorreu C.V. menor ou igual a 7\%, juntamente com sobreposição de $33 \%$, recomendada pelo fabricante.

A uniformidade da distribuição da deposição é menos influenciada pela altura da barra quando se usam menores espaçamentos entre bicos.

O programa computacional, aliado à mesa de teste, permite a obtenção de número de combinações até o limite da largura da faixa de deposição da ponta utilizada, com $5 \mathrm{~cm}$ de variações de espaçamento, mostrando-se importante ferramenta no estudo da distribuição de pontas de pulverização hidráulica em laboratório.

\section{REFERÊNCIAS BIBLIOGRÁFICAS}

AZIMI, A.H.; CARPENTER, T.G.; REICHARD, D.L. Nozzle spray distribution for pesticide application. Transactions of the ASAE, St. Joseph, v.28, p.1410-14, 1985.

CHAPPLE, A.C.; HALL, F.R.; BISHOP, B.L. Assessment of single-nozzle patternation and extrapolation to moving booms. Crop Protection, Guildford, v.12, p.207-13, 1993.

CHRISTOFOLETTI, J.C. Bicos de pulverização - seleção e uso. Diadema: Spraying Systems do Brasil, 1997. 8 p.

DOWNER, R.A.; EBERT, T.A.; THOMSON, R.S.; HALL, F.R. Herbicide spray distribution, quality and efficacy interactions: conflicts in requirements. Aspects Applied Biology, London, n.48, p.79-89, 1997.

FURLANETTI, A.C. Uniformidade de deposição de combinações de bicos em barra lateral protegida para aplicação de herbicidas. 1998. 81 f. Tese (Doutorado em Agricultura) - Faculdade de Ciências Agronômicas, Universidade Estadual Paulista, Botucatu, 1998.

GALLI, J.C.; ARRUDA, A.C. Distribuição volumétrica dos bicos pulverizadores JD14-2. Pesquisa Agropecuária Brasileira, Brasília, v.20, n.11, p.1239-44, 1985.

MATTHEWS, G. A. Pesticide application methods. 2. ed. London: Longman, 1992. 405 p.

PERECIN. D.; PERESSIN, V.A.; MATUO, T.; BARBOSA, J.C.; PIO, L.C.; BRAZ, B.A. Padrões de distribuição obtidos com bicos Twinjet em função da altura e do espaçamento entre bicos. Engenharia Agrícola, Campinas, v.14, p.19-30, 1994.

PERECIN, D.; PERESSIN, V.A.; MATUO, T.; BRAZ, B.A.; PIO, L.C. Padrões de distribuição obtidos com bicos TF-4, TJ60-11006 e TQ15006 em mesa de prova. Pesquisa Agropecuária Brasileira, Brasília, v.33, p.175-82, 1998.

SHARP, D.B. A rapid method of spray deposit measurement and its use in new apple orchards. In: BRITISH INSECTICIDE AND FUNGICIDE CONFERENCE, 7., 1973, Brighton. Proceedings... Brighton: British Crop Protection Council, 1973. p.637-42. 